\title{
Breast Injuries Classified By Bi-Rads By Magnetic Resonance And Confirmed By Biopsy
}

\author{
Gustavo Vieira Soares De Carvalho ${ }^{1}$; João Italo Fortaleza de Melo ${ }^{1}$; Luiz Dias Dutra ${ }^{1}$; Raphael Oliveira Ramos \\ Franco Netto ${ }^{2}$; Victor Augusto Ramos Fernandes ${ }^{2,3}$; Rebecca Corrêa Manfredini ${ }^{1}$; Kayque Wellek Delgado do \\ Amaral ${ }^{1}$; Messias Villa Mendonça ${ }^{1}$; Fabio Rocha ${ }^{1}$; Juliana de Almeida Rodrigues Franco Netto ${ }^{2}$; Grissom Soares \\ de Carvalho ${ }^{1}$
}

${ }^{1}$ Cerdil- Center for Radiology and Diagnostic Imaging- Dourados - Brazil

${ }^{2}$ Tissue Morphology Laboratory-FMJ-Faculty of Medicine of Jundiaí-Jundiaí-Brazil

${ }^{3}$ N. Sra. Do Patrocínio University Center - Cruzeiro do Sul University

Corresponding author: Raphael Oliveira Ramos Franco Netto; raphael_fn@hotmail.com

Received 17 July 2020;

Accepted 02 August 2020;

Published 10 August 2020

\begin{abstract}
Objective: To evaluate the malignancy or benignity of breast lesions through MRI, and its confirmation through biopsy. Materials and methods: Retrospective unicentric study. 166 patients were selected, however only 72 were included in the study, as their exams had a histopathological report compatible with a specific laboratory, responsible for analyzing the biopsies. Patients underwent MRI for breast investigation with injection of paramagnetic contrast medium, submitted to biopsy and with histopathological results. All participants were categorized by BiRADS through MRI exams. For statistical analysis, the Wilcoxon, Pearson's correlation and Chi-square tests were used. The confidence level used in the analyses was $95 \%$. Results: The most frequent histological type was Invasive Ductal Carcinoma. The BI-RADS category with the highest occurrence was 2 (two). According to the analysis of malignancy given by histopathological findings, 56.9\% (41 patients) of the lesions were malignant and $43.1 \%$ (31) were benign. When we analyzed the frequency of BI-RADS, in the categories with possibility of malignancy, we observed a distribution, BI-RADS 3 (three) with 8.3\% (6 patients), BI-RADS 4 (four) with 9.7\% ( 7 patients) and BI-RADS 5 (five) with $15.3 \%$ (11 patients), adding up to a total of $33.3 \%$ of potentially malignant lesions. Conclusion: It was observed in this study that the correlation between BI-RADS and neoplasia in the study population was weak, being categorized with a probably benign BI-RADS result was not a factor that ruled out the occurrence of cancer. Biopsy and histopathological analysis proved to be useful, demonstrating the importance of always performing it, and correlated with image findings more comprehensively.
\end{abstract}

Keywords: Bi-rads; breast lesions; biopsy; MRI

\section{Introduction}

Breast cancer (BC) is the most common câncer among women worldwide, accounting for $16 \%$ of all female cancers. Its death rate was $13.22 / 100,000$ women in 2017. Although this cancer is considered a disease in the developed world, the majority (69\%) of deaths from this cause are registered in developing countries 1 . In Brazil, breast cancer has a higher incidence in all regions. For the year 2020, 66,280 new cases were estimated, which represents an incidence rate of 43.74 cases per 100,000 women ${ }^{[1]}$.

Conservative surgery today is still considered the standard approach in early stage breast cancer, as it has a proven benefit in conjunction with radiotherapy, providing a survival rate similar to that of radical mastectomy, as long as there is adequate selection of patients. Magnetic resonance imaging (MRI) of the breasts is foregrounded in this scenario, as it has sensitivity above $90 \%$ and it is superior to conventional imaging methods in measuring the tumor and identifying additional lesions, allowing the identification of additional foci in 12.0-31, 2\% of patients in the ipsilateral breast and in $3-10 \%$ in the contralateral breast, modifying the treatment in up to one third of the patients with breast cancer ${ }^{[2]}$.

It is important to highlight that breast cancer, due to its particularities, cannot be analyzed in isolation as it presents different histological and molecular subtypes and has differences in image, prognosis and therapeutic response. It is important that studies evaluating breast MRI be individualized for each subtype, in order to define in which scenarios the imaging method performs best ${ }^{[4,5]}$.

The Breast Imaging Reporting and Data System (BIRADS), developed by the American College of Radiology since 1992, is an important guide for the standardization of breast imaging exam reports (mammography, ultrasound and MRI) and for auditing services that use these methods. Its objective is to standardize the nomenclature of the reports, which aims at the diagnostic conclusion and at proposing the conduct, according to the probability of malignancy and are currently divided into BI- 
RADS 1,2 3, 4 and 5, even though BIRADS 5 can only be described with the finding of malignancy by histological analysis $[6,8]$.

This subdivision may be even more important in MRI to define the selection of patients who need histological confirmation in cases where the lesions are not characterized by other methods, since MRI-guided biopsy is still an expensive and poorly available procedure. In addition, knowing the likelihood of malignancy of the MRI findings can assist in the correlation between imaging and anatomopathological characteristics, suggesting the need to continue the investigation through surgical resection of the lesions or confirmed by less invasive methods such as percutaneous biopsy [6,8]. Thus, the objective of this study was to evaluate the correlation between the indications proposed by BIRADS via MRI in terms of malignancy or benignity, and its confirmation through biopsy.

\section{Materials and methods}

It was a single-center retrospective study in which 166 patients were selected, who had breast MRI reports in a radiology and imaging diagnosis center. From these 166 selected, only 72 patients were included in the study, as their exams had a compatible histopathological report through a specific laboratory responsible for analyzing the biopsies. All the samples were obtained from thick needle biopsies. In this study, the following exclusion criteria were applied: patients with breast MRI without the Bi-RADS category, patients who did not have MRI performed in a high-field device, and patients with bilateral mastectomy with benign breast lesions and lesions that did not present conclusive histopathological result.

Patients underwent MRI for breast investigation with injection of paramagnetic contrast dye medium, submitted to biopsy and with a histopathological result. All participants were categorized by Bi-RADS through MRI scans. The exploratory analysis of the data was described through the calculation of measures (mean, standard deviation, minimum, median, maximum, frequency and percentage) and construction of graphs. The comparison between the levels of $\mathrm{Y}$ and $\mathrm{X}$ was performed using the Wilcoxon non-parametric test. Pearson's statistical tes ChiSquare were also used. The confidence level used in the analyzes was $95 \%$.

\section{Results}

166 exams were selected by the service's database (PACS), of which, 72 patients only met the criteria of this research. The mean age of the patients was $49.2 \pm 10.1$, ranging from 24 to 72 years (Table 1). According to the analysis of malignancy given by histopathological findings, $56.9 \%$ (41 patients) of the lesions were malignant and $43.1 \%$ (31) were benign (Table 3 and figure 6). It was observed that the Bi-RADS category with the highest occurrence was the 2 with $48.61 \%$ (35 patients) (Table 2). When analyzing the distribution between categories with potential malignancy, Bi-RADS 3 presented 8.3\% (6 patients), Bi-RADS 4 9.7\% (7 patients) and Bi-RADS 5 with $15.3 \%$ (11 patients), adding $33.3 \%$ with malignant potential to be confirmed (table 2). Ductal carcinoma was the most prevalent histological type of breast cancer with $45.45 \%$ (30 patients) (Table 4).

Table 1: Frequent age variations in the studied population.

\begin{tabular}{|l|l|l|l|l|l|l|}
\hline $\begin{array}{l}\text { Vari } \\
\text { able }\end{array}$ & $\begin{array}{l}\text { Total } \\
\text { Count }\end{array}$ & $\begin{array}{l}\text { Mea } \\
\text { n }\end{array}$ & $\begin{array}{l}\text { Standard } \\
\text { Deviation }\end{array}$ & $\begin{array}{l}\text { Minimu } \\
\text { m }\end{array}$ & $\begin{array}{l}\text { Med } \\
\text { ian }\end{array}$ & $\begin{array}{l}\text { Maxi } \\
\text { mun }\end{array}$ \\
\hline Age & 72 & 49.4 & 10.1 & 24 & 50 & 72 \\
\hline
\end{tabular}

Table 2: Percentage of patients with benign and malignant neoplasia, confirmed by biopsy

\begin{tabular}{|l|l|l|}
\hline Lesion & N & \% \\
\hline Benign & 31 & 43.06 \\
\hline Malignant & 41 & 56.94 \\
\hline Total & 72 & 100.00 \\
\hline
\end{tabular}

Table 3: Numerical and percentage distribution of BI-RADS categories in the studied population.

\begin{tabular}{|l|l|l|}
\hline BI-RADS Category & $\mathbf{N}$ & $\mathbf{\%}$ \\
\hline 0 & 3 & 4.17 \\
\hline 1 & 8 & 11.11 \\
\hline 2 & 35 & 48.61 \\
\hline 3 & 6 & 8.33 \\
\hline 4 & 7 & 9.72 \\
\hline 5 & 11 & 15.28 \\
\hline 6 & 2 & 2.78 \\
\hline Total & $\mathbf{7 2}$ & 100.00 \\
\hline
\end{tabular}

Table 4: Distribution in absolute numbers and percentage of histological types of breast cancer.

\begin{tabular}{|l|l|l|}
\hline Histological Type & N & \% \\
\hline Invasive ductal carcinoma & 30 & 45.45 \\
\hline Cyst & 7 & 10.61 \\
\hline Fibroadenoma & 6 & 9.09 \\
\hline Epithelial proliferative lesions & 4 & 6.06 \\
\hline Invasive mammary carcinoma & 4 & 6.06 \\
\hline Ductal carcinoma in situ & 3 & 4.55 \\
\hline Invasive lobular carcinoma & 3 & 4.55 \\
\hline Simple mammary adenosis & 2 & 3.03 \\
\hline Steatonecrosis & 1 & 1.52 \\
\hline Unspecified benign & 1 & 1.52 \\
\hline Ductal hyplerplasia & 1 & 1.52 \\
\hline Nuclear mammary carcinoma & 1 & 1.52 \\
\hline Intraductal papilloma & 1 & 1.52 \\
\hline Lipoma & 1 & 1.52 \\
\hline Adipose hyperplasia & 1 & 1.52 \\
\hline Total & 72 & 100.00 \\
\hline
\end{tabular}

When correlating the BI-RADS RM and the Histological Type, the $\mathrm{CP}$ was 0.201 showing a null correlation between the BI-RADS graduation and the occurrence of the histological types, not being statistically significant with p-value 0.111 . Table 5 shows that there is no correlation between the two variables. When assessed through Wilcoxon parameter the histological type confidence intervals (CI) individually, were found $(95 \% \mathrm{CI}=[5 \% ; 6.5 \%])$, indicating a very short range and consequently less variance between the data. The same analysis was performed for the BIRADS where $(95 \% \mathrm{CI}=[2 \% ; 3 \%])$ was found, reinforcing the consistency of the data.

Mann-Whitney test was also performed, in order to assess whether there were differences between the Histological Type; BIRADS RM. So there was a statistically significant difference with a p-value of 0.000 with a $95 \%$ CI. This data indicates that even though the variables did not correlate, there was a significant difference between the BI-RADS graduation and the Histological Type.

Table 5: Chi-Square Test for Association: BI-RADS MRI; Malignancy

\begin{tabular}{|l|l|l|l|}
\hline BI-RADS Category & Benign & Malignant & Total \\
\hline 0 & 0 & 3 & 3 \\
\hline
\end{tabular}




\begin{tabular}{|c|c|c|c|}
\hline & 1.708 & 1.292 & \\
\hline & 1.7083 & 2.2594 & \\
\hline \multirow[t]{3}{*}{1} & 4 & 4 & 8 \\
\hline & 4.556 & 3.444 & \\
\hline & 0.0678 & 0.0896 & \\
\hline \multirow[t]{3}{*}{2} & 24 & 11 & 35 \\
\hline & 19.931 & 15.069 & \\
\hline & 0.8309 & 1.0989 & \\
\hline \multirow[t]{3}{*}{3} & 0 & 6 & 6 \\
\hline & 3.417 & 2.583 & \\
\hline & 3.4167 & 4.5188 & \\
\hline \multirow[t]{3}{*}{4} & 3 & 4 & 7 \\
\hline & 3.986 & 3.014 & \\
\hline & 0.2440 & 0.3226 & \\
\hline \multirow[t]{3}{*}{5} & 9 & 2 & 11 \\
\hline & 6.264 & 4.736 & \\
\hline & 1.1952 & 1.5807 & \\
\hline \multirow[t]{3}{*}{6} & 1 & 1 & 2 \\
\hline & 1.139 & 0.861 & \\
\hline & 0.0169 & 0.0224 & \\
\hline Total & 41 & 31 & 72 \\
\hline
\end{tabular}
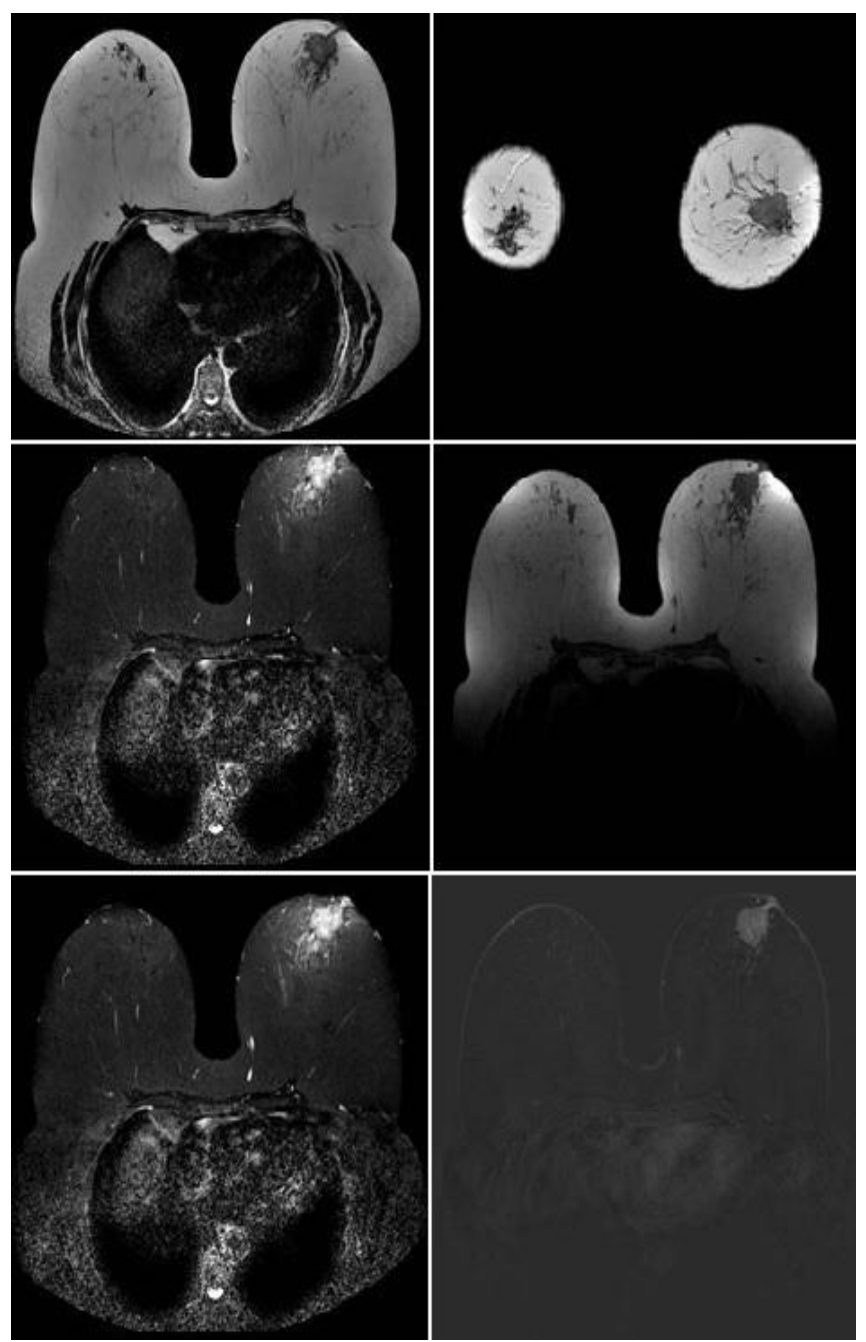

Figure 2: MRI of the Breasts - TSE (Turbo Spin Eco) T2 weighted, axial plane, TFE (Turbo Fat Spin Eco T1 weighted with dynamic sequence after injection of $20 \mathrm{ml}$ of gadolinium: Nodule with irregular margins, located in the retroareolar region of the left breast, measuring $3.0 \mathrm{~cm}$ on the longest axis, showing intense uptake of heterogeneous aspect by paramagnetic contrast. There are no signs of multifocality or multicentricity. Category V (BI-RADS).

\section{Discussion}

Magnetic resonance imaging was developed as a possible additional alternative leading to a better defined and more accurate diagnosis. The categorization of Bi-RADS categories 0,1 and 2 representing non-malignant lesions and category 3 , being lesions with a high probability of being benign, therefore biopsy is not recommended. However, the referred system advises that biopsies should not be performed on patients categorized with Bi-RADS 0, 1,2 and 3 . This procedure is often performed in a relatively large number of cases. It is considered that the most frequent reasons for biopsy are patient anxiety, lack of security of medical professionals and some factors considered to be risky for BC 9 .

Since the intention is to bring diagnostic support information to mastologists, ensuring patients who really need a biopsy are well selected, there are many attempts to establish a cutoff point that indicates the ideal category for biopsy. When searching in the literature for studies that help to compare our data, we found some in particular ${ }^{[9]}$.

One study found a predominance of benign results in BIRADS class 3 patients, in the analysis by MRI in $100 \%$ of the cases ${ }^{[5]}$. In the study, class 3 was reached by MRI for $100 \%$ of the malignant results and none of the benign ones. Among BI-RADS class 4 patients, the aforementioned study found that cases of histopathological malignancy increased progressively, in the MRI analysis, $30.7 \%$ It was not possible to observe this fact in the findings, in class $4,57 \%$ of cases were malignant, which represents a decline in malignant cases when compared to class 3 . On the other hand, the cases of histopathological benignity were, in class 4 of BI-RADS, $69.2 \%$ in the mentioned study, in this work $43 \%$ of the sample were benign cases, so there was a predominance of malignant cases in this class.

While evaluating BI-RADS in this study, class 5, a progressive increase in malignancy in MRI was observed, representing $92.8 \%$. It is noted that $18.1 \%$ of confirmed malignancies showed up in this category, representing a decline from the previous category. Furthermore, the cases of histopathological benignity increased in the study, constituting $81.8 \%$ of the patients in class 5 and the referenced study found $7.1 \%$. That is, the higher the degree of Bi-RADS in the present study, the lower the correspondence with malignancy, the same was observed with benign cases, they were much more present in the upper classes of Bi-RADS.

Another study identified the Bi-RADS categories according to the MRI parameters in the detection of malignant tumors. It was observed a VPN equal to $100 \%$ for categories 2 and 3 and notably $98.04 \%$ for category 1 , showing that there is still a possibility of FN occurring in this method ${ }^{[10]}$.

However, the persistently high NPV in the negative categories raises questions about the systematic necessity to perform biopsies in those patients. Category 4 presented a PPV of $87.5 \%$ for malignancy, which reinforces the recommendation of histological correlation as the only option, while category 5 exhibited a PPV equal to $100 \%$ for malignancy, alerting for high suspicion of invasive carcinomas when evaluating the diagnosis of malignant neoplasm. It is necessary to keep the histopathological type in mind and interpret the imaging findings more comprehensively ${ }^{[10,11]}$.

In one study, a group of researchers evaluated a group of 55 patients by MRI. Those findings were classified as BI-RADS 1 
and 2 with 19 cases, BI-RADS 3 with 10 cases, BI-RADS 4 with 13 cases and BI-RADS 5 with 13 cases as well ${ }^{[12]}$.

Regarding the correlation of histology in BI-RADS by MRI in categories 1 and 2, histopathology revealed 16 benign and 3 malignant lesions in category 3, there were 6 benign lesions and 4 malignant, in category 4 , there were 5 benign lesions (an atypical ductal hyperplasia , two typical ductal hyperplasias, a sclerosing adenosis and a fibrosis and adenosis) and 8 malignant lesions, already in category 5, were 2 benign lesions (an atypical ductal hyperplasia and a typical ductal hyperplasia) and 11 malignant lesions ${ }^{[12]}$.

Comparing these findings in categories 1 and 2, histopathology revealed 28 benign and 19 malignant lesions in category 3 there were 0 benign and 6 malignant lesions, in category 4 , there were 3 benign lesions and 4 malignant lesions, in category 5 , there were 9 benign lesions and 2 malignant lesions. In category 0 , there were 3 malignant and 0 benign lesions.

\section{Conclusion}

It was observed in this study that the correlation between BI-RADS and neoplasia in the study population was weak: being categorized as a probably benign BI-RADS it was not a factor that ruled out the occurrence of cancer. Biopsy and histopathological analysis were shown useful, demonstrating the importance of always performing them in correlation with image findings in a more comprehensive way. In the studied sample, BI-RADS was not a good predictor for neoplasia. No correlations were found between demographic variables and the data obtained in this research. Therefore, it is important to carry out further studies on the subject addressed in this work.

\section{List of abbreviations}

Breast cancer (BC)

Magnetic resonance imaging (MRI)

Breast Imaging Reporting and Data System (BI-RADS)

Picture Archiving and Communication System (PACS)

Pearson's correlation coefficient (CP)

Negative predictive value (VPN and NPV)

Positive predictive value (PPV)

False Negative (FN)

Turbo Spin Eco (TSE)

Weighting Magnetic resonance imaging(T2)

Weighting Magnetic resonance imaging (T1)

Turbo Fat Spin Eco (TFE)

\section{Data Availability}

All data in qualitative character, used by our work, as well as spreadsheets, images and others that support the results and conclusions of this study are readily available through the corresponding author.

\section{Conflicts of Interest}

The authors have no conflict of interest to declare.

\section{Funding Statement}

There was no financial support from any public or private institution for this research. The activities of this research were conducted using the authors in partnership.

\section{Authors' contributions}

The initial proposal was made by the GVSC and approved by all authors after submitting the research topic to a wide discussion and a better reformulation. JIFM conducted the literature review and collected all relevant data. KWDA and RORFN performed the data analysis. The Manuscript was written by GVSC, revised by all authors who agreed with its content and made the collective decision to submit it for consideration and possible publication

\section{Acknowledgments}

The authors of this study wish to officially thank the CERDIL Group's technical, operational and administrative team, especially Dr. Luiz Dutra, chief radiologist. To everyone in the pathological anatomy laboratory in the person of Dr. Fabio Rocha. We appreciate all the help during the period of data collection and execution of the works.

\section{References}

[1] C. De Oliveira, R. S. Pessoa, A. M. C. De Carvalho, and R. D. L. B. Magalhães, "Riskandprotectionfactors for women'shealth in thepreventionof cervical cancer," Rev. da Rede Enferm. doNord., vol. 15, no. 2, pp. 240-248, 2014.

[2] Knuttel FM, Menezes GL, van den Bosch MA, et al. Current clinical indications for magnetic resonance imaging of the breast. J Surg Oncol. 2014;110:26-31.

[3] França LKL, Bitencourt AGV, Paiva HLS, et al. Role of magnetic resonance imaging in the planning of breast cancer treatment strategies: comparison with conventional imaging techniques. Radiol Bras. 2017;50:76-81. 5. Berg WA, Gutierrez L, NessAiver MS, et al.

[4] Diagnostic accuracy of mammography, clinical examination, US, and MR imaging in preoperative assessment of breast cancer. Radiology. 2004;233:830 49. 6 .

[5] Tsina G, Simon P. Breast magnetic resonance imaging and its impact on the surgical treatment of breast cancer. Obstet Gynecol Int. 2014;2014:632074

[6] D'Orsi CJ, Sickles EA, Mendelson EB, et al. ACR BIRADS ${ }^{\circledR}$ Atlas, Breast Imaging Reporting and Data System. 5th ed. Reston, VA: American College of Radiology; 2013.

[7] Koch H. Audit in breast imaging diagnosis unit [Editorial]. Radiol Bras. 2014;47(2):

[8] Badan GM, Roveda Júnior D, Ferreira CAP, et al. Complete internal audit of a mammography service in a reference institution for breast imaging. Radiol Bras. 2014;47:74-8.

[9] Gillian MN.MR Imaging of Ductal Carcinoma In Situ. MagnResonImagingClin N Am 18 (2010) 225-240 doi:10.1016/j.mric.2010.02.004.

[10] Badan GM et al. Predictivevalues of BI-RADS ${ }^{\circledR}$ magneticresonanceimaging (MRI) inthedetection of breast ductal carcinoma in situ (DCIS).EurJour of $\begin{array}{llll}\text { Radiology } & 85 & \text { (2016) } & 1701-1707\end{array}$ http://dx.doi.org/10.1016/j.ejrad.2016.07.010. 
[11] XiaoYT, Tomohiro Y, Makiko H, Nobue K, Yutaka T, Shinobu M, Histopathologicalcharacteristics of breast ductal carcinoma in situ and associationwithimagingfindings. BreastCancer. 2016.
[12] Cilotti A, et al. Contrast-enhanced MR imaging in patientswith BI-RADS 3-5 microcalcifications. Radiolmed (2007) 112:272-286. 